Tendências em Matemática Aplicada e Computacional, 2 (2001), 43-52.

Sociedade Brasileira de Matemática Aplicada e Computacional.

\title{
Intervals as a Domain Constructor
}

R. CALLEJAS-BEDREGAL, Departamento de Matemática, Universidade Federal da Paraíba, 58059-900 João Pessoa, PB, Brasil

B.R. CALLEJAS BEDREGAL, Laboratório de Lógica e Inteligência Computacional, Departamento de Informática e Matemática Aplicada, Universidade Federal do Rio Grande do Norte, 59072-970 Natal, RN, Brasil.

\begin{abstract}
In this work we use an interval constructor on posets which when applied to a poset $\mathbf{D}$ gives a new poset whose elements are intervals of $\mathbf{D}$. We study this interval constructor from a categorical viewpoint: we verify some domain categories which are closed under this constructor. We also use this constructor in order to generalize some usual notions of the Moore interval theory, such as the interval extension of a continuous real function.
\end{abstract}

\section{Introduction}

Domain theory [15] studies a class of appropriate spaces to obtain semantic functions to give denotational semantics of programming languages. Several mathematical structures had been proposed as models for denotational semantics (semantic domains) of programming languages. Basically, a mathematical structure needs to verify the following five properties in order to be considered a semantic domain [6]: 1) it must be closed under the desired operators (product, function space, etc.), 2) canonical solutions to recursive equations must exist, 3) it must define an intuitive and flexible computability theory, 4) it must be natural to motivate and simple to describe and 5) it must have the desired basic data types.

Most of semantic domains are partially ordered sets, which can be classified in algebraic or continuous. Several cartesian closed categories of algebraic domains have been employed in the semantics of computation, and they have also been used to represent classical spaces in mathematics in an effective framework. In recent years, a new direction for application of continuous domains in computation in these spaces had emerged. Continuous domains are considered the natural setting for continuous mathematics since the representations they provide are more direct and straightforward than those of algebraic domains.

R. Moore [12] developed an interval mathematics in order to allow the control of errors in numeric computations in the solution of problems concernig real numbers. Both algebraic and continuous domains were used to represent real intervals.

Dana Scott [15] was the first to consider the poset of bounded and closed subintervals of the real line, ordered by reverse inclusion. Acióly [2], based in this interval 
poset developed a computational foundation to the Moore real interval theory. Nevertheless, the Moore theory not only comprehend real intervals, but also complex intervals, matrix and array of real and complex intervals, etc.

The concept of the set of intervals of a poset is old [5, 3]. The work of [3] uses the set of intervals of a poset with a coherent relation as primitive objects (a web) of a coherent space. Therefore, they don't use it as a categorical interval constructor on the poset category, which is the basic set up of this work.

In this work, we associate to a poset $\mathbf{D}$, a new poset $\mathbb{I}(\mathbf{D})$ whose elements are the intervals of $\mathbf{D}$ and the order is the information order based on the order of $\mathbf{D}$. We will introduce some new domain categories, such as d-algebraic and d-Scott, which take in account a domain and it opposite domain as part of the objects. We will prove that this domain categories are closed under this interval constructor.

\section{The Interval Mathematics}

We will denote by $\mathbb{I}(\mathbb{R})$ the set $\left\{[r, s] / r, s \in \mathbb{R}\right.$ e $\left.r \leq_{\mathbb{R}} s\right\}$ called the real intervals set. Each interval could be seen as a set $\left([r, s]=\left\{x \in \mathbb{R} / r \leq_{\mathbb{R}} x \leq_{\mathbb{R}} s\right\}\right)$ or as an ordered pair. The Moore theory guarantees that all interesting constructions on intervals can be obtained through their extremes.

There are several orders which can be defined on $\mathbb{I}(\mathbb{R})$ :

1. The Kulish-Miranker order $[\mathbf{9}, \mathbf{1 0}]:[a, b] \sqsubseteq[c, d] \Leftrightarrow a \leq_{\mathbb{R}} c$ and $b \leq_{\mathbb{R}} d$

2. The Moore order $[12,4]:[a, b] \leq_{M}[c, d] \Leftrightarrow[a, b]=[c, d]$ or $b \leq_{\mathbb{R}} c$

3. The set-theoretical order: $[a, b] \leq_{S}[c, d] \Leftrightarrow c \leq_{\mathbb{R}} a$ and $b \leq_{\mathbb{R}} d$

4. The information order $[15,2]:[a, b] \leq_{I}[c, d] \Leftrightarrow a \leq_{\mathbb{R}} c \leq_{\mathbb{R}} d \leq_{\mathbb{R}} b$.

\section{The Interval Constructor}

Notice that both the real intervals and the order on the set of real intervals depends upon the usual order on the real numbers. In this paper we shall consider any partially order set to formalize an interval constructor on the category POSET.

Definition 1 Let $\mathbf{D}=\langle D, \leq\rangle$ be a poset. The poset $\mathbb{I}(\mathbf{D})=\langle\mathbb{I}(D)$,

- $\mathbb{I}(D)=\{[a, b] / a, b \in D$ and $a \leq b\}$

- $[a, b] \sqsubseteq_{I}[c, d] \Leftrightarrow a \leq c$ and $d \leq b$

is called the poset of intervals of $\mathbf{D}$.

There are two natural functions from $\mathbb{I}(D)$ to $D$, which are the left and right projections $l: \mathbb{I}(D) \longrightarrow D$ and $r: \mathbb{I}(D) \longrightarrow D$ respectively, defined by

$$
l([a, b])=a \text { and } r([a, b])=b .
$$


Clearly, the function $l$ is monotonic and therefore it is a morphism from the poset $\mathbb{I}(\mathbf{D})$ to the poset $\mathbf{D}$, but the function $r$ is not monotonic. But this could be overcome by reverting the order on $\mathbf{D}$.

Definition 2 Let $\mathbf{D}=\langle D, \leq\rangle$ be a poset. The reverse poset of $\mathbf{D}$, denoted by $\mathbf{D}^{o p}$, is the pair $\mathbf{D}^{o p}=\left\langle D^{o p}, \leq_{o p}\right\rangle$, where $D^{o p}=D$ and $x \leq_{o p} y \Leftrightarrow y \leq x$.

Clearly, every poset $\mathbf{D}$ has a reverse poset and the function $r: \mathbb{I}(D) \longrightarrow D^{o p}$ is monotonic.

\section{Reversible dcpos}

We remark that a poset $\mathbf{D}=\langle D, \leq\rangle$ is a dcpo if and only if every directed subset of $\mathbf{D}$ has a supreme in $\mathbf{D}$. It is well known that a poset $\mathbf{D}=\langle D, \leq\rangle$ is a dcpo if and only if every chain of $\mathbf{D}$ has a supreme in $\mathbf{D}[7,11,1]$.

Notice that not every reverse poset of a dcpo is a dcpo. This motivate the following definition:

Definition 3 Let $\mathbf{D}=\langle D, \leq\rangle$ be a dcpo. $\mathbf{D}$ is called reversible if $\mathbf{D}^{o p}$ is a dcpo.

Lemma 1 Let $\mathbf{D}=\langle D, \leq\rangle$ be a reversible dcpo. The poset $\mathbb{I}(\mathbf{D})=\langle\mathbb{I}(D)$, $\rangle$ is a dcpo.

Proof. Let $\Delta \subseteq \mathbb{I}(D)$ be a chain. Since the projections $l: \mathbb{I}(D) \longrightarrow D$ and $r: \mathbb{I}(D) \longrightarrow D^{o p}$ are monotonic, the sets $l(\Delta) \subseteq D$ and $r(\Delta) \subseteq D$ are chains in $\mathbf{D}$ and $\mathbf{D}^{o p}$, respectively. Since $\mathbf{D}$ is a reversible dcpo every chain in $\mathbf{D}$ and $\mathbf{D}^{o p}$ has supreme. Let $x=\bigsqcup l(\Delta)$ in $\mathbf{D}$ and $y=\bigsqcup_{o p} r(\Delta)$ in $\mathbf{D}^{o p}$. Clearly, $[x, y]=\bigsqcup \Delta$ in $\mathbb{I}(\mathbf{D})$. Therefore, $\mathbb{I}(\mathbf{D})$ is a dcpo.

Proposition 1 Let $\mathbf{D}=\langle D, \leq\rangle$ be a reversible dcpo. Then $\mathbb{I}(\mathbf{D})=\langle\mathbb{I}(D)$, $\rangle$ is a reversible dcpo.

Proof. By lemma 1 it remain to prove that $\mathbb{I}(\mathbf{D})^{o p}$ is a dcpo. Let $\Delta \subseteq \mathbb{I}(D)^{o p}$ be a chain. Since the projections $l: \mathbb{I}(D)^{o p} \longrightarrow D^{o p}$ and $r: \mathbb{I}(D)^{o p} \longrightarrow D$ are monotonic, the sets $l(\Delta) \subseteq D^{o p}$ and $r(\Delta) \subseteq D$ are chains in $\mathbf{D}^{o p}$ and $\mathbf{D}$, respectively. Since $\mathbf{D}$ is a reversible dcpo, every chain in $\mathbf{D}$ and $\mathbf{D}^{o p}$ has supreme. Let $x=\bigsqcup_{o p} l(\Delta)$ in $\mathbf{D}^{o p}$ and $y=\bigsqcup r(\Delta)$ in $\mathbf{D}$. Clearly, $[x, y]=\bigsqcup_{o p} \Delta$ in $\mathbb{I}(\mathbf{D})^{o p}$. Therefore, $\mathbb{I}(\mathbf{D})^{o p}$ is a dcpo.

Corollary 1 Let $\mathbf{D}=\langle D, \leq\rangle$ be a reversible dcpo. Then the projections $l: \mathbb{I}(D) \longrightarrow D$ and $r: \mathbb{I}(D) \longrightarrow D^{o p}$ are continuous.

Proof. Let $\Delta$ be a directed set in $\mathbb{I}(\mathbf{D})$. Since, $\mathbb{I}(\mathbf{D})$ is a dcpo, $l(\Delta)$ and $r(\Delta)$ are directed set we have that $\bigsqcup \Delta, \sqcup l(\Delta)$ and $\bigsqcup_{o p} r(\Delta)$ there exist. It is enough to prove that

$$
\bigsqcup \Delta=\left[\bigsqcup l(\Delta), \bigsqcup_{o p} r(\Delta)\right]
$$


Let $\bigsqcup \Delta=[x, y]$. It is clear that $\bigsqcup l(\Delta) \leq x$ and $\bigsqcup_{o p} r(\Delta) \leq_{o p} y$. Thus, $\bigsqcup l(\Delta) \leq x \leq y \leq \bigsqcup_{o p} r(\Delta)$. Therefore,

$$
\left[\bigsqcup l(\Delta), \bigsqcup_{o p} r(\Delta)\right] \sqsubseteq[x, y] .
$$

The other inequality is clear.

Proposition 2 Let $\mathbf{D}_{1}=\left\langle D_{1}, \leq_{1}\right\rangle$ and $\mathbf{D}_{2}=\left\langle D_{2}, \leq_{2}\right\rangle$ be reversible dcpos. Then $\mathbf{D}_{1} \times \mathbf{D}_{2}$ is a reversible dcpo and $\mathbb{I}\left(\mathbf{D}_{1} \times \mathbf{D}_{2}\right) \cong \mathbb{I}\left(\mathbf{D}_{1}\right) \times \mathbb{I}\left(\mathbf{D}_{2}\right)$.

Proof. Trivially we have that $\left(\mathbf{D}_{1} \times \mathbf{D}_{2}\right)^{o p} \cong \mathbf{D}_{1}^{o p} \times \mathbf{D}_{2}^{o p}$. Therefore, since the category of dcpo is cartesian closed we have that $\mathbf{D}_{1} \times \mathbf{D}_{2}$ is reversible.

We will prove that $\mathbb{I}\left(\mathbf{D}_{1} \times \mathbf{D}_{2}\right) \cong \mathbb{I}\left(\mathbf{D}_{1}\right) \times \mathbb{I}\left(\mathbf{D}_{2}\right)$. Define the following functions: $f: \mathbb{I}\left(D_{1} \times D_{2}\right) \longrightarrow \mathbb{I}\left(D_{1}\right) \times \mathbb{I}\left(D_{2}\right)$ and $g: \mathbb{I}\left(D_{1}\right) \times \mathbb{I}\left(D_{2}\right) \longrightarrow \mathbb{I}\left(D_{1} \times D_{2}\right)$ by

$$
f([(a, b),(c, d)])=([a, c],[b, d]) \text { and } g([a, c],[b, d])=[(a, b),(c, d)] .
$$

Clearly, $f$ and $g$ are well defined. On the other hand,

$$
\begin{aligned}
{[(a, b),(c, d)] \sqsubseteq[(\widetilde{a}, \widetilde{b}),(\widetilde{c}, \widetilde{d})] } & \Leftrightarrow(a, b) \leq(\widetilde{a}, \widetilde{b}) \leq(\widetilde{c}, \widetilde{d}) \leq(c, d) \\
& \Leftrightarrow a \leq \widetilde{a} \leq \widetilde{c} \leq c \text { and } b \leq \widetilde{b} \leq \widetilde{d} \leq d \\
& \Leftrightarrow[a, c] \sqsubseteq[\widetilde{a}, \widetilde{c}] \text { and }[b, d] \sqsubseteq[\widetilde{b}, \widetilde{d]} \\
& \Leftrightarrow([a, c],[b, d]) \sqsubseteq([\widetilde{a}, \widetilde{c}],[\widetilde{b}, \widetilde{d}]) .
\end{aligned}
$$

Hence $f$ and $g$ are monotonics.

Clearly, $f \circ g=i d$ and $g \circ f=i d$. Thus $f$ is monotonic and bijective whose inverse is monotonic. Therefore, $f$ is an isomorphism.

Proposition 3 Let $\mathbf{D}_{1}=\left\langle D_{1}, \leq_{1}\right\rangle$ and $\mathbf{D}_{2}=\left\langle D_{2}, \leq_{2}\right\rangle$ be reversible dcpos. Let $f, g: D_{1} \longrightarrow D_{2}$ be continuous functions such that $f \sqsubseteq g$ (i.e., $f(x) \leq_{2} g(x)$ for each $\left.x \in D_{1}\right)$. Then the function $F: \mathbb{I}\left(D_{1}\right) \longrightarrow \mathbb{I}\left(D_{2}\right)$ defined by $F([a, b])=[f(a), g(b)]$ is the unique continuous function which makes the following diagrams:

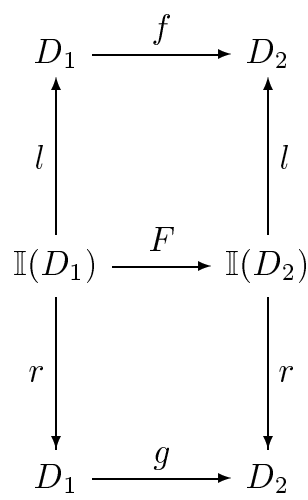

commutative. 
Proof. It is clear that $F$ is well defined and makes commutative the above diagrams. Notice that,

$$
\begin{aligned}
{[a, b] \sqsubseteq_{1}[c, d] } & \Leftrightarrow a \leq_{1} c \leq_{1} d \leq_{1} b \\
& \Leftrightarrow f(a) \leq_{2} f(c) \leq_{2} f(d) \leq_{2} g(d) \leq_{2} g(b) \\
& \Leftrightarrow[f(a), g(b)] \sqsubseteq_{2}[f(c), g(d)] \\
& \Leftrightarrow F([a, b]) \sqsubseteq_{2} F([c, d]) .
\end{aligned}
$$

Therefore, $F$ is monotonic.

We will prove next that $F$ preserve supreme of directed sets. In fact, let $\Delta \subseteq \mathbb{I}\left(D_{1}\right)$ be a directed set. We showed in corollary 1 that $\bigsqcup \Delta=\left[\bigsqcup l(\Delta), \bigsqcup_{o p} r(\Delta)\right]$. Therefore,

$$
\begin{aligned}
F(\bigsqcup \Delta) & =F\left(\left[\bigsqcup l(\Delta), \bigsqcup_{o p} r(\Delta)\right]\right) \\
& =\left[f(\bigsqcup l(\Delta)), g\left(\bigsqcup_{o p} r(\Delta)\right)\right] \\
& =\left[\bigsqcup f(l(\Delta)), \bigsqcup_{o p} g(r(\Delta))\right] \text { (f and } g \text { are continuous) } \\
& =\left[\bigsqcup l(F(\Delta)), \bigsqcup_{o p} r(F(\Delta))\right] \text { (by commutativity) } \\
& =\bigsqcup F(\Delta) .
\end{aligned}
$$

We should prove uniqueness of $F$. If $G: \mathbb{I}\left(D_{1}\right) \longrightarrow \mathbb{I}\left(D_{2}\right)$ is a continuous function such that $f \circ l=l \circ G$ and $g \circ r=r \circ G$ then,

$$
\begin{aligned}
G([a, b])=[c, d] & \Leftrightarrow c=l \circ G([a, b]) \text { and } d=r \circ G([a, b]) \\
& \Leftrightarrow c=f \circ l([a, b]) \text { and } d=g \circ r([a, b]) \\
& \Leftrightarrow c=f(a) \text { and } d=g(b) .
\end{aligned}
$$

Therefore, $G([a, b])=[f(a), g(b)]=F([a, b])$.

The above proposition generalizes the notion of interval extension of a real function. For a detailed discussion on interval extensions of real functions we refer to $[12,13]$.

\section{1. d-Consistently Completes depos}

Recall that a dcpo $\mathbf{D}$ is consistently complete if every non empty subset of $\mathbf{D}$ which has an upper bound in $\mathbf{D}$ (i.e. it is consistent) has a supreme in $\mathbf{D}$.

Definition 4 Let $\mathbf{D}=\langle D, \leq\rangle$ be a reversible dcpo. $\mathbf{D}$ is d-consistently complete if $\mathbf{D}$ and $\mathbf{D}^{o p}$ are consistently completes.

Proposition 4 Let $\mathbf{D}=\langle D, \leq\rangle$ be a reversible dcpo d-consistently complete. Then $\mathbb{I}(\mathbf{D})$ is d-consistently complete.

Proof. Let $S$ be a consistent subset in $\mathbb{I}(\mathbf{D})$. Therefore, there is $[x, y] \in \mathbb{I}(D)$ such that $S \sqsubseteq[x, y]$. Since the projections $l: \mathbb{I}(D) \longrightarrow D$ and $r: \mathbb{I}(D) \longrightarrow D^{o p}$ are monotonic we have that $l(S) \leq x$ and $r(S) \leq_{o p} y$. Thus $l(S) \subseteq D$ and $r(S) \subseteq D^{o p}$ are consistents.

Since $\mathbf{D}$ is d-consistently complete, we have that $\alpha=\bigsqcup l(S) \in D$ and $\beta=\bigsqcup_{o p} r(S) \in D^{o p}$. We will prove that $[\alpha, \beta]=\bigsqcup S$. 
Since $l(S) \leq x$ and $r(S) \leq_{o p} y$, then $\sqcup l(S) \leq x$ and $\bigsqcup_{o p} r(S) \leq_{o p} y$. Thus, $\bigsqcup l(S) \leq x \leq y \leq \bigsqcup_{o p} r(S)$.

Therefore, $[\alpha, \beta] \in \mathbb{I}(D)$. On the other hand

$$
\begin{aligned}
{[a, b] \in S } & \Rightarrow a \in l(S) \text { and } b \in r(S) \\
& \Rightarrow a \leq \bigsqcup l(S) \text { and } b \leq_{o p} \bigsqcup_{o p} r(S) \\
& \Rightarrow a \leq \bigsqcup l(S) \leq \bigsqcup_{o p} r(S) \leq b \\
& \Rightarrow[a, b] \sqsubseteq[\alpha, \beta] .
\end{aligned}
$$

Consequently $S \sqsubseteq[\alpha, \beta]$ (i.e. $[\alpha, \beta]$ is an upper bound of $S$ ). Suppouse that $[c, d]$ is another upper bound of $S$ then

$$
\begin{aligned}
{[a, b] \sqsubseteq[c, d] \forall[a, b] \in S } & \Rightarrow a \leq c \leq d \leq b, \forall[a, b] \in S \\
& \Rightarrow a \leq c \text { and } b \leq_{o p} d \forall[a, b] \in S \\
& \Rightarrow l(S) \leq c \text { and } r(S) \leq \leq_{o p} d \\
& \Rightarrow \alpha \leq c \text { and } \beta \leq_{o p} d \\
& \Rightarrow \alpha \leq c \leq d \leq \beta \\
& \Rightarrow[\alpha, \beta] \sqsubseteq[c, d] .
\end{aligned}
$$

Therefore, $[\alpha, \beta]=\bigsqcup S$. Consequently $\mathbb{I}(\mathbf{D})$ is consistently complete.

We should prove that $\mathbb{I}(\mathbf{D})^{o p}$ is consistently complete. Let $S \subseteq \mathbb{I}(D)^{o p}$ be a consistent set. Thus, there is $[x, y] \in \mathbb{I}(D)$ such that $S \sqsubseteq_{o p}[x, y]$. Therefore,

$$
\begin{aligned}
{[a, b] \sqsubseteq_{o p}[x, y] \forall[a, b] \in S } & \Rightarrow[x, y] \sqsubseteq[a, b] \quad \forall[a, b] \in S \\
& \Rightarrow x \leq a \leq b \leq y \quad \forall[a, b] \in S \\
& \Rightarrow y \leq_{o p} b \leq_{o p} a \leq_{o p} x \quad \forall[a, b] \in S .
\end{aligned}
$$

Consequently $l(S) \leq_{o p} x$ and $r(S) \leq y$. Since D is d-consistently complete and $l(S) \subseteq D^{o p}$ and $r(S) \subseteq D$ have upper bounds, we have that there exist

$$
\alpha=\bigsqcup_{o p} l(S) \in D^{o p} \text { and } \beta=\bigsqcup r(S) \in D .
$$

We will prove that $[\alpha, \beta]=\bigsqcup_{o p} S$. Clearly, $[\alpha, \beta] \in \mathbb{I}(D)$ and

$$
\begin{aligned}
{[a, b] \in S } & \Rightarrow a \in l(S) \text { and } b \in r(S) \\
& \Rightarrow a \leq o p \alpha \text { and } b \leq \beta \\
& \Rightarrow \alpha \leq a \leq b \leq \beta \\
& \Rightarrow[\alpha, \beta] \sqsubseteq[a, b] \\
& \Rightarrow[a, b] \sqsubseteq_{o p}[\alpha, \beta] .
\end{aligned}
$$

Therefore, $S \sqsubseteq_{o p}[\alpha, \beta]$, i.e. $[\alpha, \beta]$ is an upper bound of $S$ in $\mathbb{I}(\mathbf{D})^{o p}$. The proof that $[\alpha, \beta]$ is the minimal upper bound of $S$ follows analogously to the proof of 4.1 .

Therefore, $\mathbb{I}(\mathbf{D})^{o p}$ is consistently complete and consequently $\mathbb{I}(\mathbf{D})$ is d-consistently complete. 


\section{2. d-Algebraic dcpos}

Recall that $x \in D$ is compact if for each $\Delta \subseteq D$ directed set such that $x \leq \bigsqcup \Delta$, there is $y \in \Delta$ such that $x \leq y$. We denote by $\mathbf{D}^{0}$ the set $\{x \in D / x$ is compact $\}$. Also that a dcpo $\mathbf{D}$ is algebraic is for each $x \in D$ the set $\varrho_{x}=\left\{y \in \mathbf{D}^{0} / y \leq x\right\}$ is directed and $x=\bigsqcup \mathfrak{I}_{x}$.

Definition 5 [14] Let $\mathbf{D}$ be a dcpo and $B \subseteq D$. $B$ is a subbasis of $\mathbf{D}$ if for every $x \in D, \downarrow_{B} x=\{y \in B / y \leq x\}$ is a directed set and $x=\bigsqcup \downarrow_{B} x$.

Lemma 2 Let $\mathbf{D}$ be a dcpo and $B$ a subbasis of $\mathbf{D}$. Then $\mathbf{D}^{0} \subseteq B$.

Proof. Let $x \in \mathbf{D}^{0}$. Since $B$ is a subbasis of $\mathbf{D}$, we have that $\downarrow_{B} x$ is a directed set and $x=\bigsqcup \downarrow_{B} x$. Since $x$ is compact there is a $y \in \downarrow_{B} x$ (i.e. $y \in B$ and $y \leq x$ ) such that $x \leq y$. Therefore, $x=y$.

Definition 6 Let $\mathbf{D}=\langle D, \leq\rangle$ be a reversible dcpo. $\mathbf{D}$ is $\mathbf{d}$-algebraic if $\mathbf{D}$ and $\mathrm{D}^{o p}$ are algebraic.

Proposition $\mathbf{5}$ Let $\mathbf{D}$ be a d-algebraic dcpo.

1. The set $\mathbb{I}\left(\mathbf{D}^{0}\right)=\left\{[a, b] \in \mathbb{I}(D) / a, b \in D^{0}\right\}$ is a subbasis for $\mathbb{I}(\mathbf{D})$.

2. The set $\mathbb{I}\left(\left(\mathbf{D}^{o p}\right)^{0}\right)=\left\{[a, b] \in \mathbb{I}\left(D^{o p}\right) / a, b \in\left(D^{o p}\right)^{0}\right\}$ is a subbasis for $\mathbb{I}(\mathbf{D})^{o p}$.

Proof. 1) Let $B=\mathbb{I}\left(\mathbf{D}^{0}\right)$ and $[x, y] \in \mathbb{I}(D)$. Since $D$ is d-algebraic, $\rho_{x}=\left\{z \in \mathbf{D}^{0} / z \leq x\right\}$ and $\mathcal{l}_{o p} y=\left\{z \in \mathbf{D}^{o p} / z \leq_{o p} y\right\}$ are directed sets and $\sqcup \downarrow_{x}=x$ and $\bigsqcup_{o p} \downarrow_{o p} y=y$. Then $\downarrow_{B}[x, y]=\left\{[a, b] \in \mathbb{I}(D) / a \in \downarrow_{x}\right.$ and $\left.b \in \uparrow_{o p} y\right\}$.

We will show that $\downarrow_{B}[x, y]$ is a directed set in $\mathbb{I}(\mathbf{D})$ and $\bigsqcup \downarrow_{B}[x, y]=[x, y]$.

If $[a, b],[c, d] \in \downarrow_{B}[x, y]$ then $a, c \in \perp_{x}$ and $b, d \in \downarrow_{\text {op }} y$. Since $\downarrow_{x}$ and $\mathcal{\perp}_{o p} y$ are directed sets there are $u \in \complement_{x}$ and $v \in \downarrow_{\text {op }} y$ such that $a \leq u, c \leq u, b \leq_{o p} v$ and $d \leq_{o p} v$. Since $u \leq x$ and $v \leq y$, we have that

$$
a \leq u \leq x \leq y \leq b \text { and } b \leq_{o p} v \leq_{o p} y \leq_{o p} x \leq_{o p} a .
$$

Thus, $a \leq u \leq v \leq b$ and therefore $[a, b] \sqsubseteq[u, v] \in \downarrow_{B}[x, y]$. Consequently $\downarrow_{B}[x, y]$ is a directed set.

Clearly, $[x, y]$ is an upper bound of $\downarrow_{B}[x, y]$. Suppouse that $[u, v] \in \mathbb{I}(D)$ is another upper bound of $\downarrow_{B}[x, y]$. Then for each $[a, b] \in \downarrow_{B}[x, y],[a, b] \sqsubseteq[u, v]$. That is for each $[a, b] \sqsubseteq[x, y],[a, b] \sqsubseteq[u, v]$. Thus if $a \leq x$ and $b \leq_{o p} y$ then $a \leq u$ and $b \leq_{o p} v$. Therefore, $x \leq u$ and $y \leq_{o p} v$ and consequently $[x, y] \sqsubseteq[u, v]$. Hence, $[x, y]$ in the least of the upper bounds of $\downarrow_{B}[x, y]$, i.e. $[x, y]=\bigsqcup \downarrow_{B}[x, y]$.

2) Follows by the same token as 1).

Corollary $\mathbf{2}$ Let $\mathbf{D}$ be a d-algebraic dcpo. Then

1. $\mathbb{I}(\mathbf{D})^{0}=\mathbb{I}\left(\mathbf{D}^{0}\right)$.

2. $\left(\mathbb{I}(\mathbf{D})^{o p}\right)^{0}=\mathbb{I}\left(\left(\mathbf{D}^{o p}\right)^{0}\right)$. 
Proof. 1) Suppouse that $a \in D$ and $b \in D^{o p}$ are compacts. Let $\Delta \subseteq \mathbb{I}(D)$ be a direct set such that $[a, b] \sqsubseteq \bigsqcup \Delta$. We know that $\bigsqcup \Delta=[\alpha, \beta]$ where $\alpha=\bigsqcup l(\Delta) \in D$ and $\beta=\bigsqcup_{o p} r(\Delta) \in D^{o p}$. Since $[a, b] \sqsubseteq \bigsqcup \Delta=[\alpha, \beta]$ then $a \leq \alpha$ and $b \leq_{o p} \beta$. Since $l(\Delta) \subseteq D$ and $r(\Delta) \subseteq D^{o p}$ are directed sets and $a \in D$ and $b \in D^{o p}$ are compacts, we have that there are $x \in l(\Delta)$ and $y \in r(\Delta)$ such that $a \leq x$ and $b \leq_{o p} y$.

Since $\bigsqcup \Delta$ exist we have that $x \leq y$. Thus $a \leq x \leq y \leq b$ and therefore

$$
[a, b] \sqsubseteq[x, y] .
$$

On the other hand, because $x \in l(\Delta)$ there is $z \in D^{o p}$ such that $[x, z] \in \Delta$. Analogously, since $y \in r(\Delta)$ there is $w \in D$ such that $[w, y] \in \Delta$. Thus, because $\Delta$ is directed set, there is $[u, v] \in \Delta$ such that $[x, z] \sqsubseteq[u, v]$ and $[w, y] \sqsubseteq[u, v]$.

Therefore, $x \leq u \leq v \leq z$ and $w \leq u \leq v \leq y$. Hence $x \leq u \leq v \leq y$, i.e. $[x, y] \sqsubseteq[u, v]$. From 4.2 we have that $[a, b] \sqsubseteq[u, v]$ and $[u, v] \in \Delta$. Therefore, $[a, b]$ is compact in $\mathbb{I}(\mathbf{D})$.

Converselly, since $\mathbb{I}\left(\mathbf{D}^{0}\right)$ is a subbasis of $\mathbb{I}(\mathbf{D})$ we have by lemma 2 that $\mathbb{I}(\mathbf{D})^{0} \subseteq \mathbb{I}\left(\mathbf{D}^{0}\right)$.

2) Follows by the same token as 1 ).

Proposition 6 If $\mathbf{D}=\langle D, \leq\rangle$ is a d-algebraic dcpo then

1. $\mathbb{I}(\mathbf{D})$ is algebraic.

2. $\mathbb{I}(\mathbf{D})^{\text {op }}$ is algebraic.

Proof. 1) Let $[x, y] \in \mathbb{I}(D)$. We want to prove that $\{[x, y]$ is a directed set. Notice that

$$
\begin{aligned}
{[a, b] \in\{[x, y]} & \Leftrightarrow[a, b] \text { is compact and }[a, b] \sqsubseteq[x, y] \\
& \Leftrightarrow a \in D, b \in D^{o p} \text { are compacts and } a \leq x \leq y \leq b .
\end{aligned}
$$

Thus if $[a, b],[c, d] \in \mathcal{Q}[x, y]$ then $a, c \in D$ and $b, d \in D^{o p}$ are compacts and $a \leq x \leq y \leq b$ and $c \leq x \leq y \leq d$. Hence $a, c \in \uparrow x$ and $b, d \in \uparrow_{\text {op }} y$. Since $\mathbf{D}$ is d-algebraic then $q_{x}$ and $q_{o p} y$ are directed and $x=\bigsqcup q_{x}$ and $y=\bigsqcup_{o p} q_{o p} y$.

Therefore, there are $u \in \uparrow x$ and $v \in \uparrow_{o p} y$ such that $a \leq u, c \leq u, b \leq_{o p} v$ and $d \leq_{o p} v$. Since $u \leq x$ and $v \leq_{o p} y, u \leq v$. Thus $a \leq u \leq v \leq b$ and $c \leq u \leq v \leq d$. Consequently $[a, b] \sqsubseteq[u, v]$ and $[c, d] \sqsubseteq[u, v]$. Hence $q[x, y]$ is a directed set.

We will prove now that $[x, y]=\bigsqcup\{[x, y]$. Clearly, $[x, y]$ is an upper bound of $\{[x, y]$. Suppouse that $[u, v] \in \mathbb{I}(D)$ is another upper bound of $\{[x, y]$. Then

$$
\begin{aligned}
& {[a, b] \sqsubseteq[u, v] \text { for each }[a, b] \in\{[x, y] \Rightarrow a \leq u \leq v \leq b \text { for each }[a, b] \in\{[x, y]} \\
& \Rightarrow a \leq u \text { and } b \leq_{o p} v \text { for each }[a, b] \in q[x, y] \\
& \Rightarrow l(q[x, y]) \leq u \text { and } r(q[x, y]) \leq_{o p} v \\
& \Rightarrow q_{x} \leq u \text { and } q_{o p} y \leq{ }_{o p} v \\
& \Rightarrow x \leq u \text { and } y \leq_{o p} v \\
& \Rightarrow x \leq u \leq v \leq y \\
& \Rightarrow[x, y] \sqsubseteq[u, v] .
\end{aligned}
$$


Hence $[x, y]$ is the least upper bound of $\bigsqcup\{[x, y]$, i.e. $[x, y]=\bigsqcup \mathfrak{q}[x, y]$. Therefore, $\mathbb{I}(\mathbf{D})$ is algebraic.

2) Follows by the same token as 1).

Corollary $\mathbf{3}$ If $\mathbf{D}=\langle D, \leq\rangle$ is a d-algebraic dcpo then $\mathbb{I}(\mathbf{D})$ is d-algebraic.

\section{3. dcpos d-Scott}

Recall that a dcpo is a Scott dcpo if it is consistently complete and algebraic.

Definition $7 A$ dcpo $\mathbf{D}$ is a d-Scott dcpo if $\mathbf{D}$ and $\mathbf{D}^{o p}$ are $S$ cott dcpos.

From the discussion above we conclude easily that if $\mathbf{D}$ is a d-Scott dcpo then $\mathbb{I}(\mathbf{D})$ is a d-Scott dcpo.

\section{Conclusions}

We defined an interval constructor on the category POSET and proved that some domain categories, which were defined here, are closed under this constructor. Since we considered the information order, we needed to work with both a poset $\mathbf{D}$ and it reverse poset, forcing us to extend the usual notion of domains to one which takes in account the above duality. This notion was first defined in [8] under the name of bicomplete dcpo but it was not explored in the above sense. This constructor allowed us to generalize usual notions in the Moore interval theory.

The importance of this domain category jointly with the interval constructor on this category is that it allows us to give denotational semantics to programming languages which use intervals as a polimorphic and parametric data type.

\section{Acknowledgements}

The authors wish to thank the referees for the comments and sugestions made on a previous version of this paper.

Resumo. Neste trabalho usamos um constructor intervalar sobre posets o qual quando aplicado a um poset $\mathbf{D}$ nos da um novo poset cujos elementos são intervalos de D. Estudamos este construtor intervalar desde um ponto de vista categórico: verificamos algumas categorias de domínios que são fechadas sob este constructor. Usamos também este construtor para generalizar algumas noções clássicas da teoria intervalar de Moore, tal como a extensão intervalar de uma função real contínua.

\section{References}

[1] S. Abramsky and A. Jung, Domain theory, in "Handbook of Logic in Computer Science" (S. Abramsky, D.M. Gabbay and T.S.E. Maimbaum, eds.), Vol. 3, Clarendon Press, 1994. 
[2] B.M. Acióly. "Computational Foundation of Interval Mathematics", Ph.D. Thesis (in Portuguese), CPGCC, UFRGS, Porto Alegre, 1991.

[3] G.P. Dimuro, A.C.R. Costa and D.M. Claudio, A bi-structured coherence space for a global representation of the system IR of real intervals, in "CIT'99: Trends in Information Technology", Proceedings of the International Conference on Information Technology, Bubhaneswar, pp. 173-178, Tata McGraw-Hill, New Delhi, 2000.

[4] G.P. Dimuro, A.C.R. Costa and D.M. Claudio, A coherent space of rational intervals for a construction of IR, Journal of Reliable Computing 6 2000, $139-178$

[5] G. Grätzer, "General Lattice Theory", Academic Press, New York, 1978.

[6] C.A. Gunter, Comparing categories of domains, in "LNCS 239", pp. 101121, Springer-Verlag, 1985.

[7] T. Iwamura, A lemma on direct sets (in japanese), Zenkoku Shijo Sugaku Danwakai 262 (1944), 107-111.

[8] A. Jung, "Cartesian Closed Categories of Domains", Volume 66 of CWI Tracts, Centrum voor Wiskunde en Informatica, Amsterdam, 1989.

[9] U.W. Kulish and W.L. Miranker, "Computer arithmetic in theory and practice", Technical Report 33658, IBM Thomas L. Watson Research Center, 1979.

[10] U.W. Kulish and W.L. Miranker, "Computer Arithmetic in Theory and Practice", Academic Press, 1981.

[11] G. Markowsky, Chain-complete p.o. sets and directed sets with applications, Algebra Universalis 6 (1976), 53-68.

[12] R.E. Moore, "Methods and Applications for Interval Analysis", SIAM, Philadelphia, 1979.

[13] P.W. de Oliveira, T.A. Diverio and D.M. Claudio, "Foundations of Interval Mathematics" (in portuguese), Instituto de Informática, UFRGS, SAGRALuzzatto, 1997.

[14] G. Plotkin, "Domains", Department of Computer Science, University of Edinburgh, 1983.

[15] D.S. Scott, Outline of a mathematical theory of computation, in " $4^{\text {th }}$ Annual Princeton Conference on Information Sciences and Systems", pp.169176, 1970. 